\title{
General practice at the cutting edge of information technology, or failing to keep pace?
}

UK GPs are well-versed in using their electronic health record (eHR) systems to improve the process of care. Holt et al report a randomised controlled trial of a within-eHR system for calculating cardiovascular risk, and alerting GPs when a patient at significant risk attended or if information required for the risk score was missing. This was effective in increasing the amount of information recorded, but did not significantly change patient-related cardiovascular outcomes over 2 years. ${ }^{1}$ This is not surprising, as we know that computerised prompts and reminders can be effective in improving the process of clinical care, and indeed may improve patient-related outcomes if the study is adequately powered. ${ }^{2}$ The interesting aspect of this study is the use of the eHR system itself to manage the allocation to study group, the intervention, and the data collection, thus enabling a $\mathrm{PhD}$ project to conduct a 77000 patient-year randomised controlled trial. This augurs well for plans to use GP systems as the cornerstone of a national research information system. ${ }^{3}$

GP data have long been at the centre of epidemiological research, with two major UK-based databases, the General Practice Research Database ${ }^{4}$ and QResearch ${ }^{5}$ providing a significant output of research. However, using systems to facilitate randomised trials is more complex, and has been achieved in this example by the close collaboration of the GP system vendor. A national system has to manage the translation of clinical concepts and automated communication both between research and clinical care and between different proprietary clinical systems. An anglo-US project, the electronic Primary Care Research Network (ePCRN) has been working in this area for several years, producing a detailed information model for primary care, and moving to pilot systems that work across different GP clinical systems to identify potential research subjects. ${ }^{6}$ There is also considerable interest at European level in the creation of an 'active learning healthcare system' able to recruit and follow patients in clinical studies (www.transformproject.eu). ${ }^{7}$

Developments in technology are affecting the clinical encounter between doctors and patients as well as research. As Ahluwalia et al point out, the ready availability of information previously held solely within the professional domain means that patients are increasingly as well as informed as their doctors. ${ }^{8}$ When I was studying for my MRCGP in 1990, I was very taken by the work of David Tuckett and colleagues who formulated the concept of the patient as an 'expert'. ${ }^{9}$ In recent years this has become a reality, as information for both GP and patient is merely a few clicks away. The computer screen is becoming more and more something I share directly with patients, not just their record, but useful internet sites for leaflets and my own efforts to look up foreign drug names or elusive differential diagnoses (hyperthyroidism as a cause of hyperpigmentation of the tongue as a recent example).

However, all is not rosy in the garden of eHealth. The other profound change in general practice has been the intensity of workload, complexity, and risk. The shift in the management of long-term conditions to primary care, the retreat of specialist medicine into greater subspecialism, and increasing expectations mean that GPs need to be increasingly expert diagnosticians. We might reasonably ask where are the ICT tools to help us in these tasks? While prescribing alerts, clinical care process prompts, and targeting of preventive care are effective in improving the quality of care, provision of diagnostic decision support. ${ }^{10}$ This is largely because the need for knowledge representation and capture to be tightly integrated with the clinical diagnostic task has been ignored. The fine-grained representation of information relevant to diagnosis, symptoms, and signs is lacking from health record systems and is too big a task for any one proprietary vendor to support, although HL7 supports these 'Detailed Clinical Models' or 'Archetypes' as they are known.

The reason the internet has been society-changing is because it is built around a ubiquitous and maintained set of standards: standards that are largely computer system and browser independent, and cross national, cultural, and linguistic barriers to make the content freely accessible. Now we are in the midst of a new ICT revolution as Web-2.0, social networking, virtual reality, video conferencing, and mobile data become commonplace. Where is the primary care informatics equivalent? Instead we are working in a world where ICT is dependent on commercial interests with no simple means of deploying informatics tools across systems. Connecting for Health promised delivery of archetypes to clinical systems in 2006, but their development requires a degree of clinical engagement on detailed informatics that has not been a feature of the national programme, instead UK GPs rely on one of four major eHR system vendors. There is a European standard (openEHR/CEN13606) that separates knowledge representation, capture, and storage from the means of delivering and managing a record system. ${ }^{11}$ If we are to support modern general practice we need to be able to preserve the integrity of data across systems, integrate with knowledge services and decision support systems, and develop generic tools that can run on any eHR system as simply as a webpage in a browser. In order to achieve that goal we must use and develop the standards that exist. The current state of public 
finances may enforce a culture in which we concentrate on building clinical engagement, development of freelyavailable tools, and application of standards to enable widespread use of these tools in existing systems.

\section{Brendan Delaney,}

Guy's and St Thomas' Charity Chair in Primary Care Research, Kings College London, London.

\section{Provenance}

Commissioned; not peer reviewed.

\section{Competing interests}

Brendan Delaney is involved in standards development for clinical research informatics, and is principal investigator of the ePCRN and TRANSFoRm projects.

\section{REFERENCES}

1. Holt TA, Thorogood M, Griffiths F, et al. Automated electronic reminders to facilitate primary cardiovascular disease prevention: randomised controlled trial. Br J Gen Pract 2010; DOI: 10.3399/bjgp10X483904.

2. Garg AX, Adhikari NK, McDonald $\mathrm{H}$, et al. Effects of computerized clinical decision support systems on practitioner performance and patient outcomes: systematic review. JAMA 2005; 293(10): 1223-1238.

3. National Institute for Health Research. Research Capability Programme. http://www.nihr.ac.uk/systems/Pages/systems_research capability_programme.aspx (accessed 9 Mar 2010).

4. Currie CJ, Peters JR, Tynan A, et al. Survival as a function of $\mathrm{HbA}(1 \mathrm{c})$ in people with type 2 diabetes: a retrospective cohort study. Lancet 2010; 375(9713): 481-489.

5. Holt TA, Stables D, Hippisley-Cox J, et al. Identifying undiagnosed diabetes: cross-sectional survey of 3.6 million patients' electronic records. Br J Gen Pract 2008; 58(548): 192-196.

6. Speedie SM, Taweel A, Sim I, et al. The Primary Care Research Object Model (PCROM): a computable information model for practice-based primary care research. J Am Med Inform Assoc 2008; 15(5): 661-670.

7. Iakovidis I, Purcarea O. eHealth in Europe: from Vision to Reality. Stud Health Technol Inform 2008; 134: 163-168.

8. Ahluwalia S, Murray E, Stevenson F, et al. 'A heartbeat moment': qualitative study of GP views of patients bringing health information from the internet to a consultation. Br J Gen Pract 2010; 60: 88-94.

9. Tuckett D, Boulton M, Olson C, Williams A. Meetings between experts. An approach to sharing ideas in medical consultations. London: Routledge, 1985.

10. Wyatt JC, Sullivan F. eHealth and the future: promise or peril? BMJ 2005; 331(7529): 1391-1393.

11. Garde S, Knaup P, Hovenga E, Heard S. Towards semantic interoperability for electronic health records. Methods Inf Med 2007; 46(3): 332-343.

DOI: 10.3399/bigp10X483869

\section{ADDRESS FOR CORRESPONDENCE}

\section{Brendan Delaney}

Guy's and St Thomas' Charity Chair in Primary Care Research, Kings College London, Department of Primary Care and Public Health Sciences, 7th Floor, Capital House, 42 Weston St, London SE1 3QD. E-mail: Brendan.Delaney@kcl.ac.uk 